\title{
Serum adiponectin predicts fracture risk in individuals with type 2 diabetes: the Fukuoka Diabetes Registry
}

\author{
Yuji Komorita $^{1,2}$ - Masanori Iwase ${ }^{1,3} \cdot$ Hiroki Fujii $^{4} \cdot$ Toshiaki Ohkuma $^{1,5}$. \\ Hitoshi Ide $^{1,6}$ - Tamaki Jodai-Kitamura ${ }^{1}$ Akiko Sumi $^{1}$ • Masahito Yoshinari ${ }^{1}$. \\ Udai Nakamura $^{1}$ - Dongchon Kang ${ }^{2} \cdot$ Takanari Kitazono $^{1}$
}

Received: 7 March 2017 / Accepted: 7 June 2017 /Published online: 18 July 2017

(C) Springer-Verlag GmbH Germany 2017

\begin{abstract}
Aims/hypothesis Serum adiponectin has been reported to impact upon fracture risk in the general population. Although type 2 diabetes is associated with increased fracture risk, it is unclear whether serum adiponectin predicts fractures in individuals with type 2 diabetes. The aim of the study was to prospectively investigate the relationship between serum adiponectin and fracture risk in individuals with type 2 diabetes.

Methods In this study, data was obtained from The Fukuoka Diabetes Registry, a multicentre prospective study designed to investigate the influence of modern treatments on the prognoses of patients with diabetes mellitus. We followed 4869 participants with type 2 diabetes (mean age, 65 years), including 1951 postmenopausal women (defined as self-reported amenorrhea for $>1$ year) and 2754 men, for a median of 5.3 years. The primary outcomes were fractures at any site and major osteoporotic fractures (MOFs).
\end{abstract}

Masanori Iwase

iwase@ intmed2.med.kyushu-u.ac.jp

1 Department of Medicine and Clinical Science, Graduate School of Medical Sciences, Kyushu University, Maidashi 3-1-1, Higashi-ku, Fukuoka 812-8582, Japan

2 Department of Clinical Chemistry and Laboratory Medicine, Graduate School of Medical Sciences, Kyushu University, Fukuoka, Japan

3 Diabetes Centre, Hakujyuji Hospital, Fukuoka, Japan

4 Centre for Cohort Studies, Graduate School of Medical Sciences, Kyushu University, Fukuoka, Japan

5 The George Institute for Global Health, University of Sydney, Sydney, NSW, Australia

6 Division of General Internal Medicine, School of Oral Health Science, Kyushu Dental University, Kitakyushu, Japan
Results During the follow-up period, fractures at any site occurred in 682 participants, while MOFs occurred in 277 participants. Age-adjusted HRs (95\% CIs) of any fracture and MOFs for $1 \mathrm{SD}$ increment in $\log _{e}$-transformed serum adiponectin were $1.27(1.15,1.40)$ and $1.35(1.17,1.55)$ in postmenopausal women and $1.22(1.08,1.38)$ and $1.40(1.15$, 1.71 ) in men, respectively. HRs ( $95 \% \mathrm{CIs}$ ) of MOFs for hyperadiponectinaemia $(\geq 20 \mu \mathrm{g} / \mathrm{ml})$ were $1.72(1.19,2.50)$ in postmenopausal women and $2.19(1.23,3.90)$ in men. The per cent attributable risk of hyperadiponectinaemia for MOFs was as high as being age $\geq 70$ years or female sex.

Conclusions/interpretation Higher serum adiponectin levels were significantly associated with an increased risk of fractures at any site and with an increased risk of MOFs in individuals with type 2 diabetes, including postmenopausal women.

Keywords Adiponectin · Cohort study $\cdot$ Fracture $\cdot$ Type 2 diabetes

$\begin{array}{ll}\text { Abbreviations } \\ \text { BCE } & \text { Bone collagen equivalents } \\ \text { BMD } & \text { Bone mineral density } \\ \text { CHD } & \text { Coronary heart disease } \\ \text { CKD } & \text { Chronic kidney disease } \\ \text { eGFR }_{\text {cys }} & \begin{array}{l}\text { Estimated glomerular filtration rates calculated } \\ \text { based on cystatin C }\end{array} \\ \text { FRAX } & \text { Fracture risk assessment tool } \\ \text { LTPA } & \text { Leisure-time physical activity } \\ \text { MET } & \text { Metabolic equivalent } \\ \text { MOF } & \text { Major osteoporotic fracture } \\ \text { PAR } & \text { Per cent attributable risk } \\ \text { uNTx } & \text { Urinary N-terminal telopeptide of type I collagen }\end{array}$




\section{Introduction}

The recent advancements in treatments for diabetes mellitus and its complications have prolonged life expectancy, resulting in the need for a better understanding of the care of older people with diabetes [1-3]. Osteoporosis develops with ageing, especially in postmenopausal women, and leads to a marked increase in fractures after 70 years of age [4]. Diabetes mellitus is an emerging risk factor for osteoporosis and fractures, although the pathogenesis appears to be multifactorial, including non-enzymatic glycation of collagen, decreased bone turnover, oxidative stress, microvascular disease, certain diabetes medications and an increased risk of falling [5, 6]. It was previously reported that bone mineral density (BMD) did not predict fractures in individuals with type 2 diabetes [7, 8] and that the WHO fracture risk assessment tool (FRAX) algorithm underestimated their fracture risk [8]. Thus, predictors of future fractures remain to be determined in individuals with type 2 diabetes.

Adiponectin released from adipose tissue has anti-inflammatory, anti-atherogenic and insulin-sensitising effects [9]. Adiponectin and its receptors are also expressed in human osteoblasts [10], suggesting that adiponectin may play a functional role in bone metabolism. It has been reported that serum adiponectin negatively correlates with BMD [11] and may be a predictor of future fractures in non-diabetic men [12-14], although inconsistent results have been reported [15]. Hypoadiponectinaemia is associated with insulin resistance, obesity and type 2 diabetes [9] but it is not known whether serum adiponectin may also predict fractures in individuals with type 2 diabetes. The present study was designed to prospectively investigate the relationship between serum adiponectin and fracture risk in individuals with type 2 diabetes.

\section{Methods}

Study participants The Fukuoka Diabetes Registry is a multicentre prospective study designed to investigate the influence of modern treatments on the prognoses of patients with diabetes mellitus. The registry includes individuals that regularly attended teaching hospitals certified by the Japan Diabetes Society or certified diabetes clinics in Fukuoka Prefecture, Japan (UMIN Clinical Trial Registry 000002627) [16]. A total of 5131 individuals with diabetes mellitus, aged 20 years or older, were registered between April 2008 and October 2010. Exclusion criteria for the registry were as follows: (1) patients with drug-induced diabetes or those undergoing corticosteroid treatment; (2) patients undergoing renal replacement therapy; (3) patients with serious diseases other than diabetes, such as advanced malignancies or decompensated liver cirrhosis and (4) patients unable to visit diabetologists regularly. After excluding 208 participants with type 1 diabetes and one individual with no serum adiponectin concentration measurement, the remaining 4922 participants were enrolled in the baseline examination. Of the baseline participants, follow-up data regarding bone fractures were not completely obtained in 53 participants ( 33 participants died, 18 participants were lost to follow-up and two participants withdrew) and thus 4869 participants (2754 men, 1951 postmenopausal women [defined as self-reported amenorrhea for $>1$ year] and 164 premenopausal women) were ultimately selected for the present study (median follow-up period, 5.3 years; follow-up rate, $97.6 \%$ ). Study approval was obtained from the Kyushu University Institutional Review Board and all participants provided written informed consent.

Clinical evaluation at baseline Information regarding diabetes duration, smoking habits, alcohol intake, leisure-time physical activity (LTPA) and history of laser photocoagulation for diabetic retinopathy, coronary heart disease (CHD) or stroke were obtained using a self-administered questionnaire. Smoking habits and alcohol intake were classified as either current use or not. BMI was calculated from body weight and height. Participants' medical records were reviewed for all medications, including pioglitazone and insulin. LTPA was assessed as metabolic equivalent (MET) hours per week using Ainsworth's methods [17]. The dietary survey, including calcium and vitamin D intakes, was conducted using a brief selfadministered diet history questionnaire regarding the frequency of 58 food items (BDHQ; Gender Medical Research, Tokyo, Japan). The validity of ranking the energy-adjusted intakes of many nutrients has been studied previously in an adult Japanese population [18].

Laboratory measurements Blood samples were collected by venepuncture, and spot urine samples were obtained. Serum total adiponectin levels were determined by latex immunonephelometry (Mitsubishi Chemical Medience, Tokyo, Japan) with the intra-assay and inter-assay coefficients $<2 \%$ and correlation with ELISA, $r=0.99$ [19]. $\mathrm{HbA}_{1 \mathrm{c}}$ levels were assessed by HPLC (Tosoh Corp., Tokyo, Japan). Cystatin $\mathrm{C}$ concentrations were measured by latex immunonephelometry (LSI Medience, Tokyo, Japan). Urinary N-terminal telopeptide of type I collagen (uNTx) levels were determined by enzyme immunoassay (Alere Medical, Tokyo, Japan) (cut-off $89.0 \mathrm{nmol}$ bone collagen equivalents $[\mathrm{BCE}] / \mathrm{mmol}$ urinary creatinine for postmenopausal women; $66.2 \mathrm{nmol} \mathrm{BCE} / \mathrm{mmol}$-urinary creatinine for men [20]). The estimated glomerular filtration rate calculated based on cystatin $\mathrm{C}\left(\mathrm{eGFR}_{\mathrm{cys}}\right)$ was determined using the equation proposed by the Japanese Society of Nephrology [21]. The eGFR $\mathrm{Gys}_{\text {c }}$ was recently reported to be a better predictor of fractures than creatinine-based estimated GFR (eGFR) [22]. 
Assessment of fractures History of fractures was obtained at enrolment, and fractures at any site were assessed annually using a self-administered questionnaire. The first fracture that occurred after enrolment was identified. Major osteoporotic fractures (MOFs) were defined as fractures of spine, hip, upper humerus and wrist. However, since it was difficult for the participants to answer the Japanese questionnaire as to the precise fracture sites in the upper extremity, we combined upper humerus and wrist fractures as arm fractures. The primary outcomes were: (1) fractures at any site and (2) MOFs. The follow-up period was calculated as the time from enrolment to the first fracture, death or the planned study ending, whichever occurred first.

Statistical analysis Premenopausal women $(n=164)$ in the full cohort were excluded from most analyses in view of small numbers. Since the distribution of adiponectin levels was skewed toward higher values, $\log _{e}$-transformed values were used for statistical analyses. The difference in the mean values or proportions of the baseline characteristics were tested by Student's $t$ test or $\chi^{2}$ test, as appropriate. Incidence of any fracture and MOF was calculated for every $2 \mu \mathrm{g} / \mathrm{ml}$ of serum adiponectin, using the person-years method and adjusted for age and sex by the direct method for all participants. Because previous studies reported that the relationship between BMI and fractures was non-linear [23], further adjustment for BMI was performed by tertiles (tertile $1<21.9 \mathrm{~kg} / \mathrm{m}^{2}$, tertile 221.9 $25.0 \mathrm{~kg} / \mathrm{m}^{2}$ and tertile $3>25.0 \mathrm{~kg} / \mathrm{m}^{2}$ for postmenopausal women; tertile $1<22.1 \mathrm{~kg} / \mathrm{m}^{2}$, tertile $22.1-24.5 \mathrm{~kg} / \mathrm{m}^{2}$ and tertile $3>24.5 \mathrm{~kg} / \mathrm{m}^{2}$ for men). Cox proportional-hazards models were used to estimate the HRs per $1 \mathrm{SD}$ increment in $\log _{e}$-transformed serum adiponectin and the results were reported as HRs with estimation of $95 \%$ CIs for serum adiponectin levels in relation to fracture. In the multivariableadjusted model, the following covariates known to be potential risk or protective factors for fractures were selected: age, sex, BMI tertile, diabetes duration, current smoking habits, current drinking habits, daily calcium intake, LTPA, HbA 1 c, eGFR $\mathrm{H}_{\mathrm{cys}}$, uNTx, history of photocoagulation, CHD, stroke or MOFs and use of pioglitazone or insulin therapy. Multivariable-adjusted Cox proportional-hazards models were also used to evaluate the HRs and $95 \%$ CIs for each risk factor, including hyperadiponectinaemia $(\geq 20 \mu \mathrm{g} / \mathrm{ml})$, age, sex, BMI tertile, current smoking habits, current drinking habits, daily calcium intake, LTPA, $\mathrm{HbA}_{1 \mathrm{c}} \geq 7.5 \%$ (58.5 mmol/mol), chronic kidney

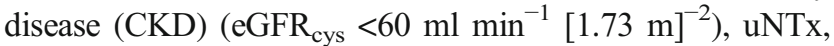
history of photocoagulation, CHD, stroke or MOFs and pioglitazone use or insulin therapy. Per cent attributable risk (PAR) was calculated as a percentage of the prevalence of fracture risk potentially attributable to a risk factor that would be reduced if the risk factor was removed. The positive and negative predictive values, sensitivity and specificity of hyperadiponectinaemia for incident fractures were determined.
To validate the prediction of hyperadiponectinaemia, the positive and negative predictive values and the sensitivity and specificity were calculated on a random sample of $50 \%$ of the total participants and then the estimated fracture number was compared with the actual fracture number for the remaining participants. All statistical analyses were performed with the Statistical Analysis Software (SAS) version 9.3 (SAS Institute, Cary, NC, USA). Values of $p<0.05$ were considered statistically significant in all analyses.

\section{Results}

Baseline characteristics of all participants, postmenopausal women and men according to the fracture group are summarised in Table 1. During the follow-up period (median 5.3 years; follow-up rate $97.6 \%$ ), fractures occurred in 682 participants including 413 postmenopausal women and 249 men. MOFs occurred in 277 participants (138 in spine, 53 in hip, 86 in arm). Fractures were more common among women than men. Participants with fractures were older and had longer duration of diabetes, higher serum adiponectin level and lower GFR $_{\text {cys }}$ and a higher percentage had a history of PC for retinopathy than participants without fractures. In postmenopausal women with fractures, a significantly increased percentage had a history of pioglitazone use, any fracture, MOFs and CHD compared with postmenopausal women without fractures. In men, $\mathrm{HbA}_{1 \mathrm{c}}$, uNTx and the percentage having used insulin were significantly higher in those with fractures.

Serum adiponectin concentration varied according to BMI tertile in postmenopausal women (tertile $1,11.8 \mu \mathrm{g} / \mathrm{ml}[95 \%$ CI 11.1, 12.7]; tertile 2, $9.9 \mu \mathrm{g} / \mathrm{ml}$ [95\% CI 9.4, 10.4]; tertile 3, $9.8 \mu \mathrm{g} / \mathrm{ml}[95 \%$ CI 9.3, 10.3]; $p$ for trend <0.001) and in men (tertile 1, $9.3 \mu \mathrm{g} / \mathrm{ml}[95 \%$ CI 9.0, 9.8]; tertile 2, $7.5 \mu \mathrm{g} / \mathrm{ml}$ [95\% CI 7.3, 7.9]; tertile 3, $6.7 \mu \mathrm{g} / \mathrm{ml}[95 \%$ CI 6.3, 7.0], $p$ for trend $<0.001$ ).

Figure 1a displays the age- and sex-adjusted incidence of any fracture and MOFs for every $2 \mu \mathrm{g} / \mathrm{ml}$ of serum adiponectin in all participants. The age- and sex-adjusted incidence of any fracture consistently increased up to 24$26 \mu \mathrm{g} / \mathrm{ml}$ of serum adiponectin. In contrast, MOFs remained stable until $18-20 \mu \mathrm{g} / \mathrm{ml}$ of serum adiponectin, at which point it rapidly increased, peaking at 24-26 $\mu \mathrm{g} / \mathrm{ml}$ of serum adiponectin. Fracture incidences did not substantially change after additional adjustment for BMI (Fig. 1b).

Table 2 displays the HRs for any fracture and MOFs per 1 SD $\log _{e}$-transformed serum adiponectin. In postmenopausal women, the age-adjusted HR (95\% CIs) was 1.27 (1.15, $1.40)$ for any fracture and $1.35(1.17,1.55)$ for MOFs. The HRs did not change substantially after adjustments for age, sex, BMI tertile, diabetes duration, current smoking habit, current drinking habit, daily calcium intake, LTPA, $\mathrm{HbA}_{1 \mathrm{c}}$, 


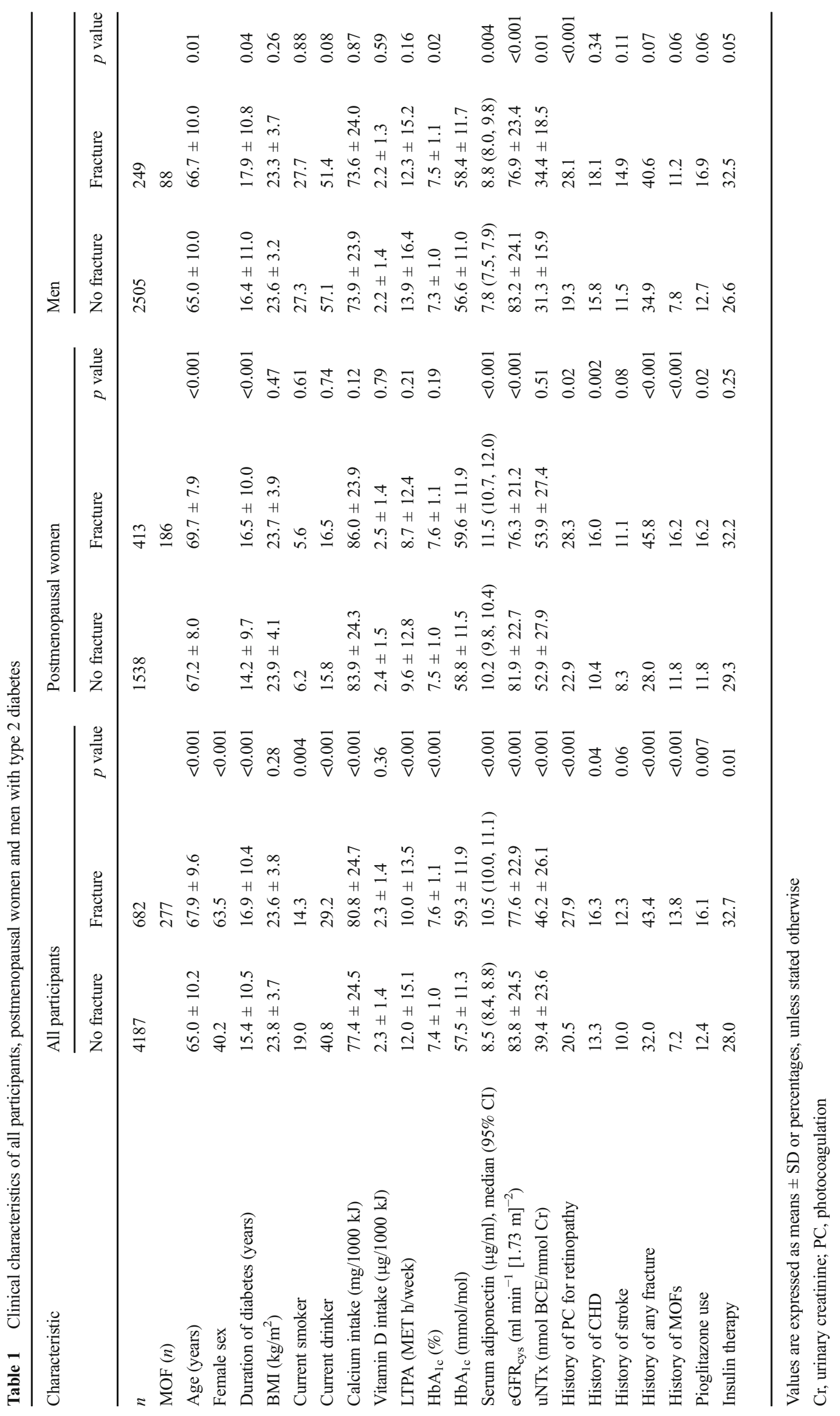




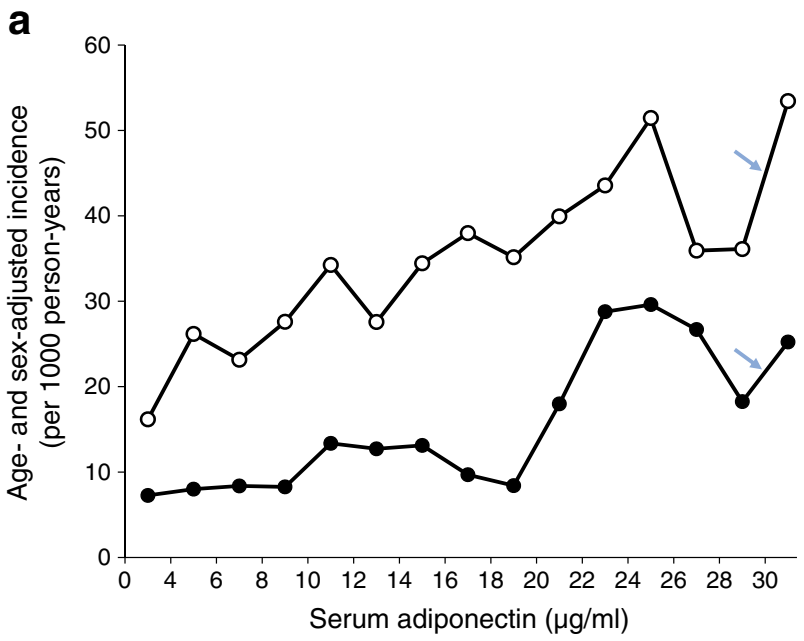

$\begin{array}{lrrrrrrrrrrrrrrr}n & 321 & 855 & 894 & 760 & 594 & 375 & 245 & 188 & 136 & 94 & 102 & 70 & 45 & 40 & 150\end{array}$ $\begin{array}{lrrrrrrrrrrrrrrr}\text { Any fracture } & 24 & 86 & 95 & 105 & 99 & 54 & 42 & 34 & 22 & 21 & 23 & 20 & 10 & 8 & 39 \\ \text { MOF } & 9 & 24 & 35 & 34 & 45 & 27 & 17 & 10 & 8 & 9 & 14 & 13 & 7 & 4 & 21\end{array}$

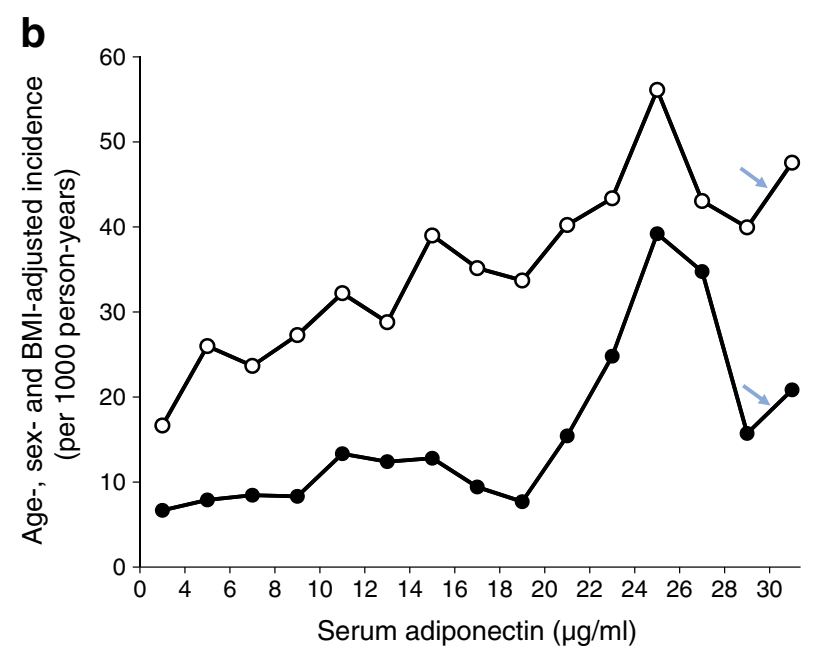

$\begin{array}{llllllllllllllll}n & 321 & 855 & 894 & 760 & 594 & 375 & 245 & 188 & 136 & 94 & 102 & 70 & 45 & 40 & 150\end{array}$ $\begin{array}{lrrrrrrrrrrrrrrr}\text { Any fracture } & 24 & 86 & 95 & 105 & 99 & 54 & 42 & 34 & 22 & 21 & 23 & 20 & 10 & 8 & 39 \\ \text { MOF } & 9 & 24 & 35 & 34 & 45 & 27 & 17 & 10 & 8 & 9 & 14 & 13 & 7 & 4 & 21\end{array}$

Fig. 1 Incidence of fractures at any site (white circles) and MOFs (black circles) for every $2 \mu \mathrm{g} / \mathrm{ml}$ of serum adiponectin in all participants with type 2 diabetes, adjusted (a) for age and sex or (b) for age, sex and BMI tertile. Blue arrows indicate incidence at $30 \mu \mathrm{g} / \mathrm{ml}$ of serum adiponectin

eGFR $_{\text {cys }}$, history of photocoagulation, CHD, stroke and MOFs and insulin use. Further adjustments for uNTx or uNTx plus pioglitazone use did not substantially alter the HRs. In men, the age-adjusted HR (95\% CIs) was 1.22 $(1.08,1.38)$ for any fracture and $1.40(1.15,1.71)$ for MOFs. Although these remained significant after adjustments for multiple variables, the significance was diminished by further adjustment for pioglitazone use.

Risk factors for fractures at any site and MOFs were evaluated in postmenopausal women and men using Cox proportional models (Table 3). Hyperadiponectinaemia was defined as adiponectin levels equal to or higher than $20 \mu \mathrm{g} / \mathrm{ml}$, based on incidence rates (Fig. 1) and a previous study [14]. In postmenopausal women, hyperadiponectinaemia, age and history of MOFs were associated with any fracture and MOFs. Furthermore, insulin therapy was significantly associated with MOFs. In men, age, low BMI (tertile 1 vs tertile 2) and uNTx were associated with any fracture and MOFs. History of photocoagulation for retinopathy, history of MOFs and use of pioglitazone were significantly associated with fractures at any site in men, whereas hyperadiponectinaemia, higher BMI (tertile 3 vs tertile 2) and current smoking were significantly associated with MOFs.

Table 4 shows the PAR of significant risk factors for fractures at any site and MOFs in postmenopausal women and men. In postmenopausal women, history of MOFs had the highest PAR (52.4\% for any fracture and $60.9 \%$ for MOFs). Hyperadiponectinaemia produced the second highest PAR for any fracture (37.7\%) and the third highest for MOFs (50.8\%) in postmenopausal women. In men, hyperadiponectinaemia had the highest PAR for MOFs (72.3\%). In all participants, PAR of female sex and hyperadiponectinaemia was $55.8 \%$ and $46.8 \%$ in any fracture and $64.2 \%$ and $64.7 \%$ in MOFs, respectively.

Finally, the reproducibility and usefulness of hyperadiponectinaemia as a predictor for fractures were evaluated. We determined the positive and negative predictive values as well as sensitivity and specificity on a random sample of 50\% of the total participants $(n=2435)$, and then validated the predictions on the remaining sample $(n=2434)$. For fractures at any site, the positive predictive value was $22.3 \%$, the negative predictive value was $87.9 \%$, the sensitivity was $17.2 \%$ and the specificity was $90.9 \%$ in the random sample. Estimated fracture number was 56 in participants with hyperadiponectinaemia, when the actual fracture number was 66 . For MOFs, the positive predictive value was $13.0 \%$, the negative predictive value was $95.7 \%$, the sensitivity was $25.0 \%$ and the specificity was $90.7 \%$ in the random sample. Estimated fracture number was 33 in those with hyperadiponectinaemia, when the actual fracture number was 36 .

\section{Discussion}

In this prospective study, we demonstrated that higher serum adiponectin levels were significantly associated with an increased risk of fractures at any site as well as MOFs in participants with type 2 diabetes. To our knowledge, this is the first prospective cohort study to demonstrate the association between serum adiponectin and fracture risk in individuals with diabetes. Particularly, previous studies have failed to demonstrate an association between serum adiponectin and fractures in postmenopausal women $[12,13]$. The impact of hyperadiponectinaemia as a risk factor for MOFs appears to be as strong as wellestablished risk factors, such as age $\geq 70$ years or female sex. 
Table 2 Adjusted HRs for fractures at any site or MOFs per $1 \mathrm{SD}$ $\log _{e}$-transformed serum adiponectin in postmenopausal women and men with type 2 diabetes

\begin{tabular}{|c|c|c|c|c|}
\hline \multirow[t]{2}{*}{ Adjustment } & \multicolumn{2}{|l|}{ Any fracture } & \multicolumn{2}{|l|}{ MOF } \\
\hline & $\mathrm{HR}(95 \% \mathrm{CI})$ & $p$ value & $\mathrm{HR}(95 \% \mathrm{CI})$ & $p$ value \\
\hline \multicolumn{5}{|l|}{ Postmenopausal women } \\
\hline Age adjusted & $1.27(1.15,1.40)$ & $<0.001$ & $1.35(1.17,1.55)$ & $<0.001$ \\
\hline Multivariate adjusted & $1.29(1.17,1.40)$ & $<0.001$ & $1.37(1.19,1.59)$ & $<0.001$ \\
\hline$+\mathrm{uNTx}$ adjusted & $1.27(1.15,1.40)$ & $<0.001$ & $1.37(1.18,1.59)$ & $<0.001$ \\
\hline$+\mathrm{uNTx}$, pioglitazone adjusted & $1.29(1.11,1.39)$ & $<0.001$ & $1.33(1.12,1.56)$ & $<0.001$ \\
\hline \multicolumn{5}{|l|}{ Men } \\
\hline Age adjusted & $1.22(1.08,1.38)$ & 0.002 & $1.40(1.15,1.71)$ & 0.001 \\
\hline Multivariate adjusted & $1.16(1.02,1.32)$ & 0.024 & $1.36(1.10,1.68)$ & 0.004 \\
\hline +uNTx adjusted & $1.13(1.00,1.29)$ & 0.060 & $1.30(1.05,1.61)$ & 0.016 \\
\hline$+u N T x$, pioglitazone adjusted & $1.06(0.92,1.23)$ & 0.430 & $1.16(0.90,1.48)$ & 0.250 \\
\hline
\end{tabular}

Multivariate adjustments include age, sex, BMI tertile, diabetes duration, current smoking habit, current drinking habit, daily calcium intake, $\mathrm{LTPA}, \mathrm{HbA}_{1 \mathrm{c}}, \mathrm{eGFR}_{\mathrm{cys}}$ and history of photocoagulation for retinopathy, CHD, stroke or fragility fractures and insulin therapy
It was previously reported that there was a negative correlation between serum adiponectin and BMD in general [11], although adiponectin levels did not correlate with fracture risk in elderly Swedish men [15]. In the Rancho Bernardo Study [12], however, high serum adiponectin was associated with vertebral fracture risk in 277 men (mean age, 75 years) but not in 251 postmenopausal women (mean age 76 years). In the
Health Aging and Body Composition study [13], an association between high serum adiponectin levels and nonvertebral fractures was observed in 1568 men but not in 1476 postmenopausal women (aged 70-79 years). In the Osteoporotic Fractures in Men study in Sweden [14], an association was observed between high serum adiponectin levels and fractures at any site in 999 men aged $70-81$ years. The gradient of

Table 3 Adjusted HRs of risk factors for fractures at any site or MOFs in postmenopausal women and men with type 2 diabetes

\begin{tabular}{|c|c|c|c|c|c|c|c|c|}
\hline \multirow[t]{3}{*}{ Risk factor } & \multicolumn{4}{|c|}{ Postmenopausal women } & \multicolumn{4}{|l|}{ Men } \\
\hline & \multicolumn{2}{|l|}{ Any fracture } & \multicolumn{2}{|l|}{ MOF } & \multicolumn{2}{|l|}{ Any fracture } & \multicolumn{2}{|l|}{ MOF } \\
\hline & $\mathrm{HR}(95 \% \mathrm{CI})$ & $\begin{array}{l}p \\
\text { value }\end{array}$ & $\mathrm{HR}(95 \% \mathrm{CI})$ & $\begin{array}{l}p \\
\text { value }\end{array}$ & $\operatorname{HR}(95 \% \mathrm{CI})$ & $\begin{array}{l}p \\
\text { value }\end{array}$ & $\operatorname{HR}(95 \% \mathrm{CI})$ & $\begin{array}{l}p \\
\text { value }\end{array}$ \\
\hline Hyperadiponectinaemia $(\geq 20 \mu \mathrm{g} / \mathrm{ml})$ & $1.47(1.12,1.92)$ & 0.01 & $1.72(1.19,2.50)$ & 0.004 & $1.13(0.73,1.75)$ & 0.53 & $2.19(1.23,3.90)$ & 0.01 \\
\hline Age $(/ 10$ years $)$ & $1.02(1.01,1.04)$ & $<0.001$ & $1.05(1.03,1.07)$ & $<0.001$ & $1.02(1.00,1.03)$ & 0.02 & $1.06(1.03,1.09)$ & $<0.001$ \\
\hline BMI (tertile 1 vs tertile 2 ) & $0.88(0.68,1.13)$ & 0.79 & $1.11(0.77,1.59)$ & 0.59 & $1.56(1.15,2.13)$ & 0.01 & $1.98(1.13,3.47)$ & 0.02 \\
\hline BMI (tertile 3 vs tertile 2) & $0.99(0.77,1.26)$ & 0.86 & $1.02(0.71,1.47)$ & 0.91 & $1.32(0.95,1.84)$ & 0.28 & $1.81(1.00,3.26)$ & 0.05 \\
\hline Current smoker & $0.98(0.64,1.50)$ & 0.92 & $1.63(0.93,2.86)$ & 0.09 & $1.12(0.84,1.50)$ & 0.52 & $1.64(1.01,2.65)$ & 0.05 \\
\hline Current drinker & $1.14(0.87,1.48)$ & 0.33 & $0.96(0.63,1.46)$ & 0.85 & $0.90(0.70,1.17)$ & 0.41 & $0.67(0.43,1.03)$ & 0.07 \\
\hline Calcium intake (/100 mg/1000 kJ) & $1.24(0.83,1.85)$ & 0.34 & $1.40(0.76,2.57)$ & 0.29 & $0.73(0.42,1.26)$ & 0.28 & $0.41(0.16,1.07)$ & 0.07 \\
\hline LTPA (/10 METs h/week) & $1.11(0.90,1.37)$ & 0.35 & $1.06(0.78,1.46)$ & 0.70 & $0.96(0.74,1.24)$ & 0.80 & $0.88(0.57,1.37)$ & 0.57 \\
\hline $\mathrm{HbA}_{1 \mathrm{c}} \geq 7.5 \%(58.5 \mathrm{mmol} / \mathrm{mol})$ & $1.05(0.85,1.30)$ & 0.58 & $1.12(0.82,1.52)$ & 0.50 & $1.13(0.86,1.47)$ & 0.34 & $0.78(0.48,1.25)$ & 0.30 \\
\hline $\begin{array}{l}\text { CKD }\left(\mathrm{eGFR}_{\text {cys }}<60 \mathrm{ml} \mathrm{min}^{-1}\right. \\
{\left[1.73 \mathrm{~m}^{-2}\right)}\end{array}$ & $1.17(0.90,1.52)$ & 0.20 & $1.23(0.85,1.78)$ & 0.28 & $1.31(0.94,1.84)$ & 0.10 & $1.24(0.73,2.11)$ & 0.43 \\
\hline uNTx (/10 nmol BCE/mmol Cr) & $1.02(0.99,1.06)$ & 0.32 & $1.02(0.97,1.08)$ & 0.40 & $1.10(1.03,1.18)$ & 0.01 & $1.19(1.08,1.31)$ & $<0.001$ \\
\hline History of PC for retinopathy & $1.10(0.87,1.40)$ & 0.45 & $0.98(0.69,1.40)$ & 0.92 & $1.44(1.07,1.93)$ & 0.02 & $1.23(0.74,2.03)$ & 0.43 \\
\hline History of CHD & $1.30(0.99,1.71)$ & 0.08 & $1.42(0.97,2.09)$ & 0.07 & $1.05(0.75,1.46)$ & 0.79 & $1.11(0.65,1.89)$ & 0.70 \\
\hline History of stroke & $1.01(0.73,1.38)$ & 0.70 & $1.25(0.82,1.92)$ & 0.30 & $1.15(0.80,1.65)$ & 0.49 & $1.46(0.86,2.51)$ & 0.16 \\
\hline History of MOFs & $3.03(2.19,4.19)$ & $<0.001$ & $2.06(1.41,3.02)$ & $<0.001$ & $2.11(1.15,3.88)$ & 0.05 & $1.58(0.81,3.08)$ & 0.18 \\
\hline Pioglitazone use & $1.31(0.95,1.79)$ & 0.07 & $1.35(0.84,2.17)$ & 0.21 & $1.55(1.06,2.26)$ & 0.02 & $1.83(0.98,3.45)$ & 0.06 \\
\hline Insulin therapy & $1.10(0.87,1.40)$ & 0.46 & $1.42(1.01,2.01)$ & 0.05 & $1.13(0.84,1.52)$ & 0.38 & $1.49(0.92,2.42)$ & 0.10 \\
\hline
\end{tabular}

Cr, urinary creatinine; $\mathrm{PC}$, photocoagulation 
Table 4 PAR of significant risk factors for fractures at any site or MOFs in postmenopausal women and men with type 2 diabetes

\begin{tabular}{|c|c|c|c|c|}
\hline \multirow[t]{2}{*}{ Risk factor } & \multicolumn{2}{|c|}{ Postmenopausal women } & \multicolumn{2}{|l|}{ Men } \\
\hline & Any fracture $(\%)$ & MOFs $(\%)$ & Any fracture $(\%)$ & MOFs $(\%)$ \\
\hline Hyperadiponectinaemia $(\geq 20 \mu \mathrm{g} / \mathrm{ml})$ & 37.7 & 50.8 & & 72.3 \\
\hline Age $\geq 70$ years & 34.4 & 58.5 & 34.6 & 64.8 \\
\hline BMI tertile 1 vs tertile 2 & & & 32.1 & 52.6 \\
\hline Smoking & & & & 15.1 \\
\hline uNTx $>66.2 \mathrm{nmol} \mathrm{BCE} / \mathrm{mmol} \mathrm{Cr}$ & & & 46.8 & 63.5 \\
\hline History of PC for retinopathy & & & 35.8 & \\
\hline History of MOFs & 52.4 & 60.9 & 30.1 & \\
\hline Pioglitazone use & & & 26.1 & \\
\hline Insulin therapy & & 29.4 & & \\
\hline
\end{tabular}

PAR was calculated as a percentage of the prevalence of fracture risk potentially attributable to a risk factor that would be reduced if the risk factor was removed

$\mathrm{Cr}$, urinary creatinine; $\mathrm{PC}$, photocoagulation fracture risk expressed as HR per $1 \mathrm{SD}$ of serum adiponectin was $1.30(95 \%$ CI 1.09, 1.55) after multivariable adjustment, consistent with the results of our study (HR 1.22). The Swedish study also showed that the relationship between serum adiponectin and the risk of fractures was non-linear and that the association increased above $18 \mu \mathrm{g} / \mathrm{ml}$ of serum adiponectin. In individuals with type 2 diabetes, one small cross-sectional study (231 men, 170 postmenopausal women) demonstrated that there was a positive correlation between adiponectin and vertebral fractures only in men [24]. In contrast with these previous studies, we demonstrated that serum adiponectin was a fracture risk factor in postmenopausal women. This difference may be related to the fact that Japanese individuals with type 2 diabetes are not obese (mean BMI $23.8 \mathrm{~kg} / \mathrm{m}^{2}$ in the present study); obesity typically increases BMD and may prevent fractures [25].

Since adipocytes and osteoblasts share a common progenitor [26], adiponectin may play a functional role in bone homeostasis, but its exact role remains to be elucidated. Adiponectin stimulated human osteoblast proliferation and differentiation, while adiponectin stimulated receptor activator of nuclear factor- $\mathrm{kB}$ ligand and reduced osteoprotegerin mRNA expression in human osteoblasts, leading to osteoclastogenesis [27]. However, conflicting results have been reported regarding the effect of adiponectin on bone in adiponectinknockdown or adiponectin-overexpressing mice [28, 29]. In the present study, the association between adiponectin and MOFs remains significant after adjusting for uNTx, which is a bone resorption marker. However, prospective cohort studies suggest that high serum adiponectin levels may predict difficulty in daily activities [30], lower extremity muscle strength [31] and incident falls [32]. It is possible that the association between adiponectin and incident falls may lead to an increased fracture risk, although we did not assess falls in our study.
In the present study, there was a steady increase in the risk of any fractures with increasing serum adiponectin, whereas a threshold may be present for MOFs (Fig. 1). In a previous study, wherein 406 fractures (72 vertebral and 334 nonvertebral) occurred, there was no threshold noted for serum adiponectin [13]. However, in another study of elderly individuals aged over 70 years, wherein 150 fractures (99 fractures of the spine, hip, forearm and humerus) occurred, there was a threshold at $18 \mu \mathrm{g} / \mathrm{ml}$ of serum adiponectin [14]. Assuming that the fractures in the former study are similar to any fracture and those in the latter study to MOFs in our study, our results are consistent with these previous studies. These results may be related to the possibility that any fractures may be more traumatic than MOFs. In this context, hyperadiponectinaemia was not a significant risk for any fracture in men. Regarding adiponectin and MOFs, these findings suggest that bone strength may be affected upon exposure to high concentrations of adiponectin.

The risk of MOFs was higher in women than in men, and their respective risk factors may be different. Our study showed some sex differences in the risks for MOFs. Lower and higher BMI was a significant risk for MOFs in men, whereas there was no relationship between BMI and fractures in postmenopausal women (Table 3). Furthermore, uNTx was a significant fracture risk in men but not in postmenopausal women. However, serum adiponectin was significantly associated with MOFs, even after adjustment for BMI tertile and uNTx, in both sexes (Table 2). The relationship between serum adiponectin and fracture risk remained significant after adjustment for pioglitazone use in postmenopausal women but the relationship was no longer significant in men. This difference may be explained as follows. First, the correlation between serum adiponectin and BMI may be stronger in men (Spearman's $r-0.260, p<0.001$ ) than in postmenopausal women (Spearman's $r-0.162, p<0.001$ ). Because low 
BMI was a significant risk factor for fracture in men, it might diminish the significance in men. Second, the impact of adiponectin on bone metabolism may be different between the sexes, as Ealey et al [33] showed that the negative effect of adiponectin overexpression on bone mass and strength was stronger in female mice than in male mice. Third, as the number of fractures was smaller in men than in postmenopausal women, the power to detect statistical differences may be different. Further studies are necessary to determine the mechanisms of sex differences.

Some fracture risk factors are specific to individuals with type 2 diabetes. Poor glycaemic control has been reported to be associated with increased fracture risk $[5,6]$. In our study, there were no significant differences in fracture risk between participants with $\mathrm{HbA}_{1 \mathrm{c}} \geq 7.5 \%(58.5 \mathrm{mmol} / \mathrm{mol})$ and those with $\mathrm{HbA}_{1 \mathrm{c}}<7.5 \%(58.5 \mathrm{mmol} / \mathrm{mol})$ or HR per $1.0 \%$ increase in $\mathrm{HbA}_{1 \mathrm{c}}$ (data not shown). Thiazolidinediones, including pioglitazone, have been reported to be associated with an increased risk of fractures in the limbs rather than in the spine or hip [5,34]. In the current study, fractures tended to be increased at any site in pioglitazone-treated participants. Chronic diabetic complications have been reported to be associated with fractures $[5,6]$. However, we found that only a history of photocoagulation for retinopathy was associated with any fracture risk in men (Table 3 ).

The strength of the current study lies in the prospective cohort design and the relatively large number of participants (mean age 65 years). This enabled us to include a wide range of potential confounders, including diet, physical activity, medications, uNTx and diabetic complications. However, several limitations of our study should be noted. First, fractures as an outcome were derived from self-reported data. When the accuracy of the self-administered questionnaire was evaluated in 455 fracture events by comparison with medical records, the agreement rate was $93.0 \%$ and there were no morphometric vertebral fractures. However, information regarding external forces or incident falls that caused fractures was difficult to assess by a selfadministered questionnaire. Second, we did not measure BMD and thus FRAX scoring was not available. Although the validation of hyperadiponectinaemia as a predictor for fractures was evaluated, we were unable to determine whether the addition of serum adiponectin to the scoring algorithm was favourable. Third, only total adiponectin levels were measured. Although adiponectin may function differently depending on adiponectin multimerisation [35], it has been reported that the correlation between total and high-molecular-weight adiponectin is significant [36]. Furthermore, the correlation with fractures was stronger for total adiponectin levels than high-molecular-weight adiponectin levels [23]. Finally, it remains unclear whether the conclusions of the current study can be generalised to other ethnic populations. Particularly, there may be racial or ethnic differences in serum adiponectin levels, and genetic variants do exist in the adiponectin gene [37]. Serum adiponectin levels were not elevated in a Japanese population, even though Japanese individuals had a much lower prevalence of obesity [38].

In conclusion, the present study demonstrates that serum adiponectin levels may be useful to predict future fractures in individuals with type 2 diabetes, including postmenopausal women. Further studies are required to confirm our findings in other populations.

Acknowledgements The authors thank Y. Kiyohara, Y. Doi, T. Ninomiya, S. Kanba, S. Kumagai, S. Kaizu, Y. Hirakawa, C. Matsumoto, C. Kitaoka (Kyushu University, Fukuoka, Japan), N. Tsutsu, N. Sasaki (Fukuoka Red Cross Hospital, Fukuoka, Japan), K. Nunoi, Y. Sato, Y. Uchizono, A. Yamauchi, K. Itoh, C. Kono (St Mary's Hospital, Kurume, Japan), S. Nohara, H. Imoto, K. Amano (Steel Memorial Yawata Hospital, Kitakyushu, Japan), D. Gotoh, T. Himeno, M. Toyonaga (Kyushu Central Hospital, Fukuoka, Japan), N. Shinohara, A. Tsutsumi (Fukuoka Higashi Medical Centre, Koga, Japan), Y. Idewaki, M. Nakano, M. Matsuo, S. Morimoto, T. Hyodo (Hakujyuji Hospital, Fukuoka, Japan), M. Minami (Clinic Minami Masae, Fukuoka, Japan), M. Wada (Wada Miya Naika Clinic, Fukuoka, Japan), Y. Yokomizo (Yokomizo Naika Clinic, Kitakyushu, Japan), M. Kikuchi, Y. Kikuchi (Kikuchi Naika Clinic, Itoshima, Japan), R. Nomiyama (Suzuki Naika Clinic, Fukuoka, Japan), S. Nakamura (Nakamura Naika Clinic, Fukuoka, Japan), K. Tashiro (Oshima Eye Hospital, Fukuoka, Japan), M. Yoshinari (Yoshinari Naika Clinic, Fukuoka, Japan), K. Ichikawa (Fukutsu Naika Clinic, Fukutsu, Japan) and T. Omae (Hisayama Research Institute For Lifestyle Diseases, Fukuoka, Japan). The authors also thank clinical research coordinators, C. Ohba, Y. Ono (Hisayama Research Institute for Lifestyle Diseases, Hisayama, Japan) and K. Sekioka (Kyushu University, Fukuoka, Japan) and those in the administration office, T. Matake (Hisayama Research Institute for Lifestyle Diseases, Hisayama, Japan) and J. Ishimatsu (Kyushu University, Fukuoka, Japan).

Data availability The datasets generated during and/or analysed during the current study are available from the corresponding author on reasonable request.

Funding This work was supported, in part, by the Japan Society for the Promotion of Science KAKENHI (grant numbers 23249037 and 23659353 for MI, 16K00861 for HF) from the Ministry of Education, Culture, Sports, Science and Technology of Japan.

Duality of interest The authors declare that there is no duality of interest associated with this manuscript.

Contribution statement $\mathrm{YK}$ and MI were responsible for the study concept and design. YK, MI and HF conducted the analysis and TO, HI, TJ-K, AS, MY, UN, DK and TK interpreted the data and contributed to the discussion. YK and MI drafted the manuscript. All authors participated in critically revising the manuscript and approved the final version. MI is the guarantor of this work and, as such, had full access to all the data in the study and takes responsibility for integrity of the data and the accuracy of the data analysis. 


\section{References}

1. Gregg EW, Li Y, Wang J et al (2014) Changes in diabetes-related complications in the United States, 1990-2010. N Engl J Med 370: 1514-1523

2. Cheng YJ, Imperatore G, Geiss LS et al (2013) Secular changes in the age-specific prevalence of diabetes among U.S. adults: 19882010. Diabetes Care 36:2690-2696

3. Kirkman MS, Briscoe VJ, Clark N et al (2012) Diabetes in older adults. Diabetes Care 35:2650-2664

4. Cummings SR, Melton LJ (2002) Epidemiology and outcomes of osteoporotic fractures. Lancet 359:1761-1767

5. Dede AD, Tournis S, Dontas I, Trovas G (2014) Type 2 diabetes mellitus and fracture risk. Metabolism 63:1480-1490

6. Schwartz AV (2016) Epidemiology of fractures in type 2 diabetes. Bone 82:2-8

7. Vestergaard P (2007) Discrepancies in bone mineral density and fracture risk in patients with type 1 and type 2 diabetes - a metaanalysis. Osteoporos Int 18:427-444

8. Schwartz AV, Vittinghoff E, Bauer DC et al (2011) Association of BMD and FRAX score with risk of fracture in older adults with type 2 diabetes. JAMA 305:2184-2192

9. Kadowaki T, Yamauchi T, Kubota N, Hara K, Ueki K, Tobe K (2006) Adiponectin and adiponectin receptors in insulin resistance, diabetes, and the metabolic syndrome. J Clin Invest 116:1784-1792

10. Berner HS, Lyngstadaas SP, Spahr A et al (2004) Adiponectin and its receptors are expressed in bone-forming cells. Bone 35:842-849

11. Biver E, Salliot C, Combescure C et al (2011) Influence of adipokines and ghrelin on bone mineral density and fracture risk: a systematic review and meta-analysis. J Clin Endocrinol Metab 96: 2703-2713

12. Araneta MR, von Muhlen D, Barrett-Connor E (2009) Sex differences in the association between adiponectin and BMD, bone loss, and fractures: the Rancho Bernardo study. J Bone Miner Res 24: 2016-2022

13. Barbour KE, Zmuda JM, Boudreau R et al (2011) Adipokines and the risk of fracture in older adults. J Bone Miner Res 26:1568-1576

14. Johansson H, Oden A, Lerner UH et al (2012) High serum adiponectin predicts incident fractures in elderly men: osteoporotic fractures in men (MrOS) Sweden. J Bone Miner Res 27:1390-1396

15. Michaelsson K, Lind L, Frystyk J et al (2008) Serum adiponectin in elderly men does not correlate with fracture risk. J Clin Endocrinol Metab 93:4041-4047

16. Ohkuma T, Fujii $H$, Iwase $M$ et al (2013) Impact of sleep duration on obesity and the glycemic level in patients with type 2 diabetes: the Fukuoka Diabetes Registry. Diabetes Care 36:611-617

17. Ainsworth BE, Haskell WL, Whitt MC et al (2000) Compendium of physical activities: an update of activity codes and MET intensities. Med Sci Sports Exerc 32:S498-S504

18. Kobayashi S, Honda S, Murakami K et al (2012) Both comprehensive and brief self-administered diet history questionnaires satisfactorily rank nutrient intakes in Japanese adults. J Epidemiol 22:151159

19. Nishimura A, Sawai T (2006) Determination of adiponectin in serum using a latex particle-enhanced turbidimetric immunoassay with an automated analyzer. Clin Chim Acta 371:163-168

20. Nishizawa Y, Ohta H, Miura M et al (2013) Guidelines for the use of bone metabolic markers in the diagnosis and treatment of osteoporosis (2012 edition). $\mathrm{J}$ Bone Miner Metab 31:1-15

21. Horio M, Imai E, Yasuda Y, Watanabe T, Matsuo S, Collaborators Developing the Japanese Equation for Estimated GFR (2013) GFR estimation using standardized serum cystatin C in Japan. Am J Kidney Dis 61:197-203

22. Ensrud KE, Parimi N, Fink HA et al (2014) Estimated GFR and risk of hip fracture in older men: comparison of associations using cystatin C and creatinine. Am J Kidney Dis 63:31-39

23. Compston JE, Flahive J, Hosmer DW et al (2014) Relationship of weight, height, and body mass index with fracture risk at different sites in postmenopausal women: the Global Longitudinal study of Osteoporosis in Women (GLOW). J Bone Miner Res 29:487-493

24. Kanazawa I, Yamaguchi T, Yamamoto M, Yamauchi M, Yano S, Sugimoto T (2009) Relationships between serum adiponectin levels versus bone mineral density, bone metabolic markers, and vertebral fractures in type 2 diabetes mellitus. Eur J Endocrinol 160:265-273

25. Johansson H, Kanis JA, Oden A et al (2014) A meta-analysis of the association of fracture risk and body mass index in women. J Bone Miner Res 29:223-233

26. Caplan AI (1991) Mesenchymal stem cells. J Orthop Res 9:641650

27. Luo XH, Guo LJ, Xie H et al (2006) Adiponectin stimulates RANKL and inhibits OPG expression in human osteoblasts through the MAPK signaling pathway. J Bone Miner Res 21: $1648-1656$

28. Kajimura D, Lee HW, Riley KJ et al (2013) Adiponectin regulates bone mass via opposite central and peripheral mechanisms through FoxO1. Cell Metab 17:901-915

29. Williams GA, Wang Y, Callon KE et al (2009) In vitro and in vivo effects of adiponectin on bone. Endocrinology 150:3603-3610

30. Kizer JR, Arnold AM, Strotmeyer ES et al (2010) Change in circulating adiponectin in advanced old age: determinants and impact on physical function and mortality. The Cardiovascular Health Study All Stars Study. J Gerontol A Biol Sci Med Sci 65:1208-1214

31. Huang C, Tomata Y, Kakizaki M et al (2015) High circulating adiponectin levels predict decreased muscle strength among older adults aged 70 years and over: a prospective cohort study. Nutr Metab Cardiovasc Dis 25:594-601

32. Huang $\mathrm{C}$, Momma $\mathrm{H}$, Niu $\mathrm{K}$ et al (2016) High serum adiponectin levels predict incident falls among middle-aged and older adults: a prospective cohort study. Age Ageing 45:366-371

33. Ealey KN, Kaludjerovic J, Archer MC, Ward WE (2008) Adiponectin is a negative regulator of bone mineral and bone strength in growing mice. Exp Biol Med (Maywood) 233:15461553

34. Aubert RE, Herrera V, Chen W, Haffner SM, Pendergrass M (2010) Rosiglitazone and pioglitazone increase fracture risk in women and men with type 2 diabetes. Diabetes Obes Metab 12:716-721

35. Waki H, Yamauchi T, Kamon J et al (2003) Impaired multimerization of human adiponectin mutants associated with diabetes. Molecular structure and multimer formation of adiponectin. J Biol Chem 278:40352-40363

36. van Andel M, Drent ML, van Herwaarden AE, Ackermans MT, Heijboer AC (2016) A method comparison of total and HMW adiponectin: HMW/total adiponectin ratio varies versus total adiponectin, independent of clinical condition. Clin Chim Acta 465:30-33

37. Mente A, Razak F, Blankenberg S et al (2010) Ethnic variation in adiponectin and leptin levels and their association with adiposity and insulin resistance. Diabetes Care 33:1629-1634

38. Ikezaki H, Ai M, Schaefer EJ et al (2016) Ethnic differences in glucose homeostasis markers between the Kyushu-Okinawa Population Study and the Framingham Offspring Study. Sci Rep 6:36725 